\title{
Design and Development of Plant's Growth Measurement System
}

\author{
Ramandeep Kaur \\ Lecturer in ECE (Shaheed Bhagat Singh State Technical Campus, Ferozepur, India)
}

\begin{abstract}
Growth of plant is influenced by environmental conditions and topography. So there is need to measure dynamics of plant to understand how plants respond to variation in water content and long term water conditions. Various studies have found that variation of the diameter and height of plant stem has an important correlation ship with water status, temperature, light and humidity. Here, an interface between a C3008 smart camera and a computer has been developed. This camera module has digital output and digital video port that supplies a continuous 8-16 bit range of data stream. It is used to capture the images of a plant. A MATLAB GUI has been developed for performing Sobel Edge Detection on a plant image to determine its height, maximum and minimum width. Testing of different plants is done at different locations. The results attained prove that the system is capable of measuring the changes (diameter and height changes) of plant's growth accurately.
\end{abstract}

Keywords: Digicam, Edge detection, Growth, Sobel edge detection, Stem diameter and height.

\section{Introduction}

Currently, there are various techniques for measuring stem diameter and height. Several experiments were conducted to investigate the development and expansion of plant. The conventional monitoring system used LVDT sensors in order to acquire the stem diameter has some severe shortages such as that we need to change sensors of different scale to fit the different range of diameters. We can only get the increment of the diameter while leaving the real value of the stem diameter unknown to us. The sensor may restrain the natural growth of the crops. On the other hand, strain gauges are sensors that rely on the change in gauge resistance in determining the strain within the gauge section. Measuring the growth of plant using strain gauge does not provide accurate values of plant's growth. It required large precision. The output signal is weak and unwanted signal and interference is also introduced. Thermal effect also arises in strain gauge method.

The objective of this project is to find the diameter and height of a plant. This project consists of two parts which can be divided into hardware and software. The basic structure consists of digicam module, controller and PC as shown in Fig 1.1. The system comes into an action when a user presses the button, mounted on it for the user interface. In first module, there is camera interfaced to AVR controller to acquire an image. Main advantage of this camera is that it has digital output. Digital port that supplies a continuous 8-16 bit wide range data stream. It will capture the image of plant at different interval of time. Digicam pass the data to the PC through USB. All the functions of camera contrast, brightness, intensity etc. are programmable through I2C interface. For further processing of the image, it is fed to the PC. In this module image is detected, edge detection, data acquisition and storage of data is performed.

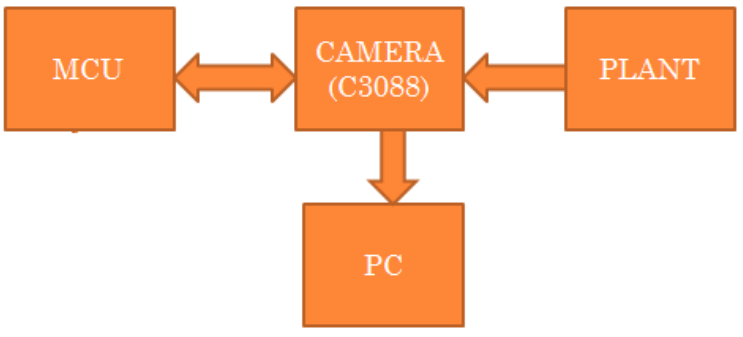

Figure 1.1 The block diagram system

\section{Related Work}

Currently, there are various techniques for measuring stem diameter and height. Several experiments were conducted to investigate the development and expansion of a plant. The results of these experiments indicate that the variation of the diameter of plant stem, height, temperature and humidity has a prominent correlation ship with the water status. The conventional monitoring system used LVDT sensors in order to acquire the stem diameter has some severe shortages such as that we need to change sensors to different scale to fit the different range of diameters. We can only get the increment of the diameter while leaving the real value 
of the stem diameter unknown to us. N.M Khairi et. al. [1] measures the stem changes using strain gauge. Strain gauges are sensors that rely on the change in gauge resistance in determining the strain within the gauge section. Measuring the growth of plant using strain gauge does not provide accurate values of plant's growth. It required large precision. The output signal is weak and unwanted signal and interference is also introduced. Thermal effects also arise in strain gauge method. Both of the above discussed method may also restrain the natural growth of the crops. Jiaxing Che et. al. [2] developed a model using computer vision and embedded systems. it was based on smart camera, computer vision technology and embedded DSP processor technology. Ariff et. al. [3] had measured Stem diameter using a digital caliper, with the readings taken midway on the stem. Heights of the samples were measured using a measurement tape, from the tip of the shoot to the ground.

\section{Algorithm Used for Develop System}

Edge detection refers to the process of identifying and locating sharp discontinuities in an image. The discontinuities are abrupt changes in pixel intensity which characterize boundaries of objects in a scene. Classical methods of edge detection involve convolving the image with an operator (a 2-D filter), which is constructed to be sensitive to large gradients in the image while returning values of zero in uniform regions. In digital image, the so-called edge is a collection of the pixels whose gray value has a step or roof change, and it also refers to the part where the brightness of the image local area changes significantly. The gray profile in this region can generally be seen as a step. That is, in a small buffer area, a gray value rapidly changes to another whose gray value is largely different with it. Edge widely exists between objects and backgrounds, objects and objects, primitives and primitives. The edge of an object is reflected by the discontinuity of the gray. Therefore, the general method of edge detection is to study the changes of a single image pixel in a gray area, use the dissimilarity of the edge neighboring first order or second-order to detect the edge. Edge detection is mainly the measurement, detection and location of the changes in gray image. Image edge is the most basic features of the image. When we observe the objects, the clearest part we see firstly is edge and line. According to the composition of the edge and line, we can know the object structure. Therefore, edge extraction is an important technique in graphics processing and feature extraction. The basic idea of edge detection is as follows: Firstly, use edge enhancement operator to highlight the local edge of the image. Then, define the pixel "edge strength" and set the threshold to extract the edge point set. However, because of the noise and the blurring image, the edge detected may not be continuous. There are various edge detection algorithms available. In this proposed work, Sobel Edge Detection algorithm has been used.

\subsection{Sobel Edge Detection}

The Sobel operator performs a 2-D spatial gradient measurement on an image and emphasizes regions of high spatial gradient that correspond to edges. In general it is used to find the approximate absolute gradient magnitude at each point in an input grayscale image. Compared to other edge operator, Sobel has two main advantages:

1. Since the introduction of the average factor, it has some smoothing effect to the random noise of the image.

2. Because it is the differential of two rows or two columns, so the elements of the edge on both sides have been enhanced, so that the edge seems thick and bright.

The operator consists of a pair of $3 \times 3$ convolution kernels as shown in Fig 3.1. Single kernel is simply the other rotated by $90^{\circ}$.

\begin{tabular}{|c|c|c|}
\hline-1 & 0 & +1 \\
\hline-2 & 0 & +2 \\
\hline-1 & 0 & +3 \\
\hline
\end{tabular}

\begin{tabular}{|c|c|c|}
\hline+1 & +2 & +1 \\
\hline 0 & 0 & 0 \\
\hline-1 & -2 & -1 \\
\hline
\end{tabular}

Figure 3.1 Masks used by sobel operator

These kernels are designed to respond maximally to edges running vertically and horizontally relative to the pixel grid, single kernel for each of the two perpendicular directions. The kernels can be applied separately to the given image, to produce separate measurements of the gradient component in each orientation (call these Gx and Gy). These can then be joint together to find the absolute magnitude of the gradient at each point and the orientation of that gradient. The gradient magnitude is given by [4]:

$$
|G|=\sqrt{G^{2}+\mathrm{Gy}^{2}}
$$

Although typically, an approximate magnitude is computed using:

This is much faster to compute.

$$
|G|=|G x|+|G y|
$$




\section{Experimental Results and Explanation}

Once the image is received at PC side from the camera for further processing edge detection and data storage functions are performed on the image. In the PC a matlab GUI has been developed for performing Sobel Edge Detection on a plant image to determine its height and width. There are the two different options are provided for the selection of a plant image. That is either we can manually cropped the image (pressing 2) or select the full image (pressing 1). Fig 4.1 shows the selection of plant image with provision of cropping by pressing 2.

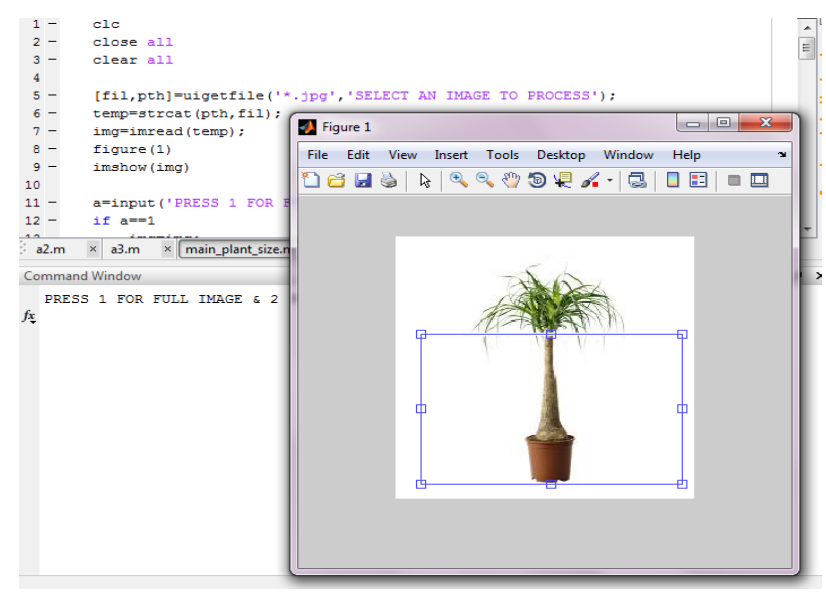

Figure 4.1 Selection of cropped image

Fig 4.2 is presenting the plant image with highlighted edges as well as the measured values of height, maximum and minimum width of a plant in terms of pixels.

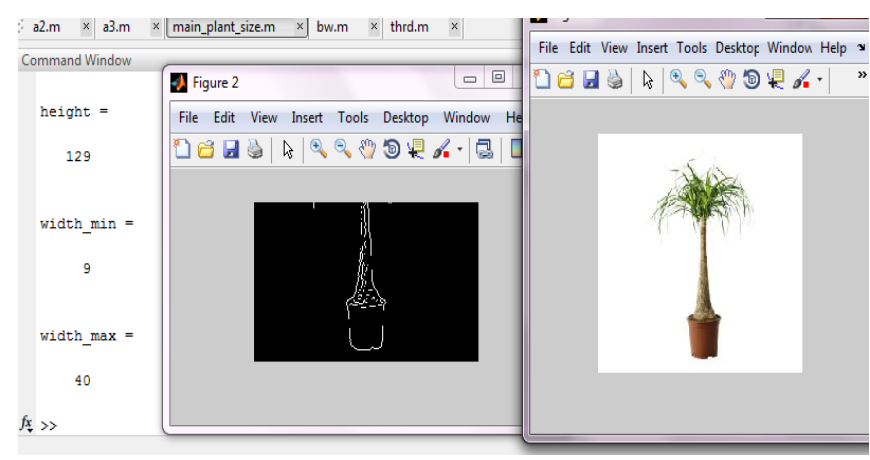

Figure 4.2 Edge detected image

Fig 4.3 shows the selection of full plant image by pressing 1 .

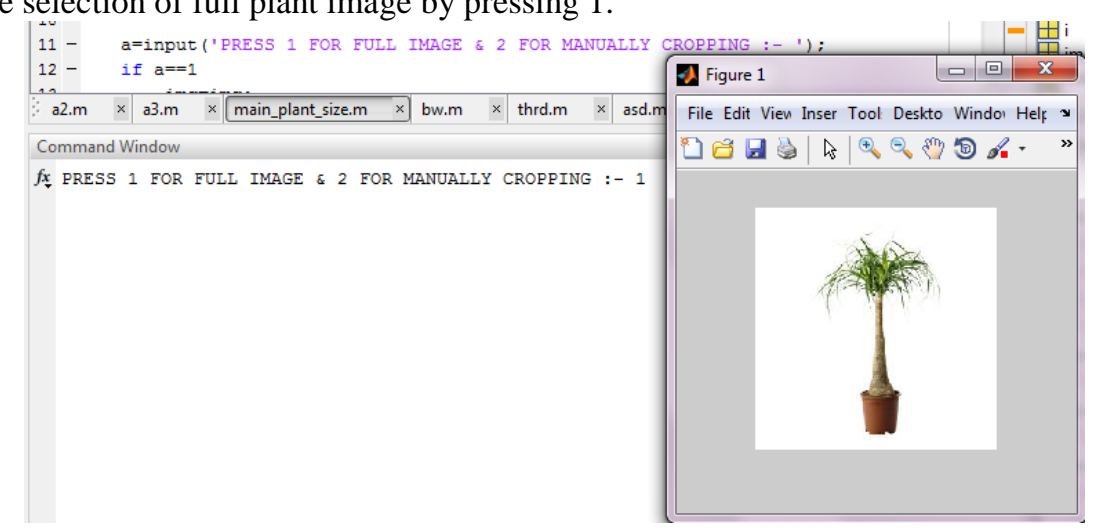

Figure 4.3 Selection of full image (pressing 1)

Fig 4.4 is presenting the full plant image with highlighted edges as well as the measured values of height and width (minimum and maximum) of a plant in terms of pixels. 


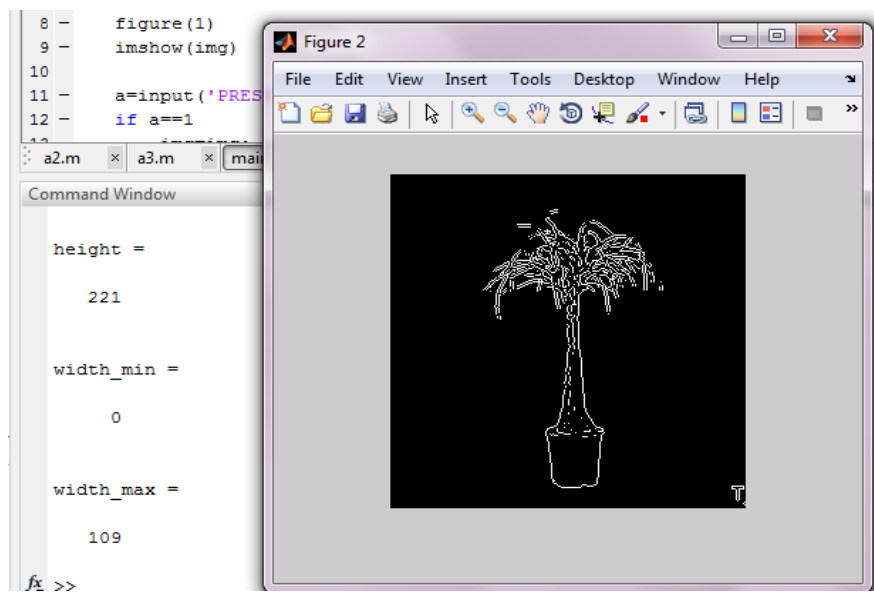

Figure 4.4 Edge detected for full plant's image

Table I Testing Of Different Plants At Different Locations

\begin{tabular}{|c|c|c|c|}
\hline FIGURE & HEIGHT IN PIXELS & MINIMUM WIDTH IN PIXELS & MAXIMUM WIDTH IN PIXELS \\
\hline (A) & 110 & 12 & 13 \\
\hline (B) & 215 & 11 & 15 \\
\hline (C) & 245 & 5 & 7 \\
\hline (D) & 240 & 10 & 13 \\
\hline
\end{tabular}

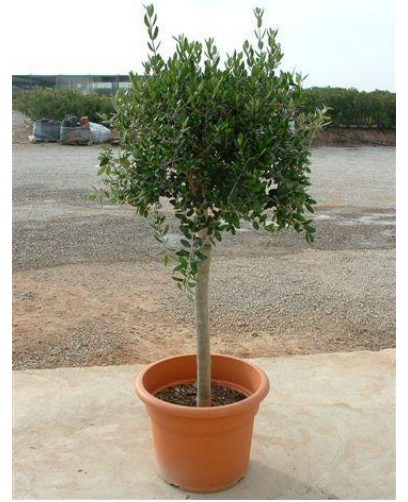

(a)

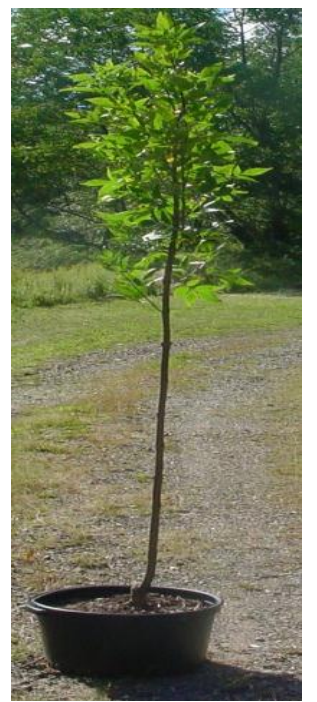

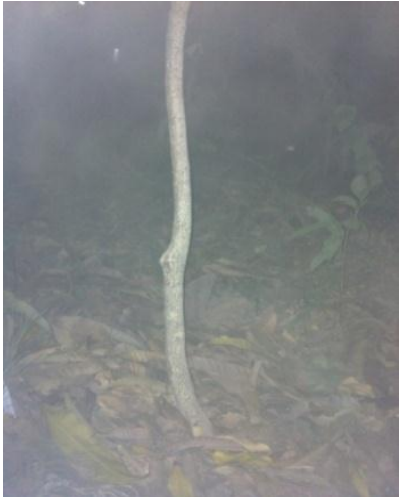

(b)

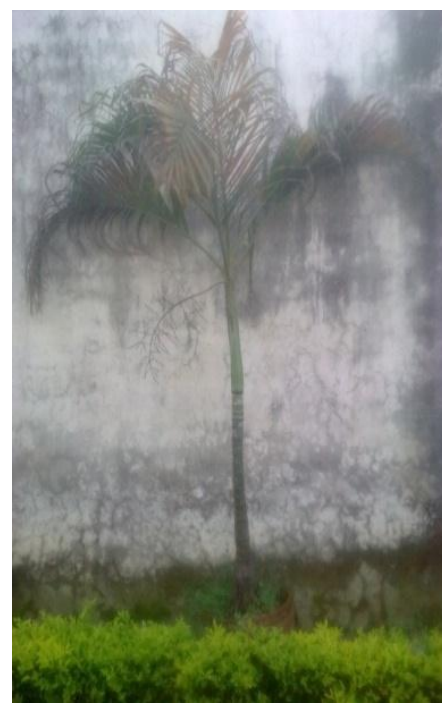

Figure 4.5 At different locations images captured by the camera 


\section{Conclusion}

In this research, growth of a plant is measured using interface between digital camera and a computer. This interface allows a user to get continuous video of the plants. Testing of different plants at different places has been done. A MATLAB GUI has been developed for performing sobel edge detection on a plant image to determine its height and width. Two different types of options for the selection of a plant image are provided. Either the full image or cropping of the image can be done. The algorithm provides height and maximum and minimum width of the plant's stem in terms of pixels.

\section{Future Scope}

As a future enhancement the system can be made wireless (mobile) and fully automatic by giving the entire control to the MCU. Advanced edge detection algorithms must be used for enhancing the accuracy of edge detection. The limitation of the system developed is that it takes large time for processing the images captured by camera. So someone has to think for the solution to fasten the response time.

\section{References}

[1]. N.M.Khairi, M.A.Marni Shah Rizam, M.S.B. Tahir, N.M. Naimah, M.I. Zainol Abidin,"Optimization of strain gauge for stem measurement using PIC based instrumentation," System Engineering and Technology (ICSET), 2011 IEEE International Conference, pp.196-199, 27-28 June 2011.

[2]. Jiaxing Che, Chunjiang Zhao, Yunhe Zhang, Cheng Wang, Xiaojun Qiao, Xinlu Zhang, "Plant stem diameter measuring device based on computer vision and embedded system," World Automation Congress (WAC), 2010, pp.51-55, 19-23 Sept. 2010.

[3]. Ariff, E.A.R.E. Suratman, M.N.Abdullah," Stomatal conductance, chlorophyll content, diameter and heightin different growth stages of rubber tree (Hevea brasiliensis) saplings," Business, Engineering and Industrial Applications (ISBEIA), 2011 IEEE Symposium, pp.84-88, 25-28 Sept. 2011.

[4]. Nick Kanopoulos, et.al. ; "Design of an Image Edge Detection Filter using the Sobel Operator", Journal of Solid State Circuits, IEEE, vol. 23, Issue: 2, pp. 358-367, April 1988.

[5]. Beant Kaur, Mr. Anil Garg, "Comparative study of different edge detection techniques" International Journal of Engineering Science and Technology (IJEST) Vol. 3 No. 3 March 2011.

[6]. Wenshuo Gao; Xiaoguang Zhang; Lei Yang; Huizhong Liu, "An improved Sobel edge detection," Computer Science and Information Technology (ICCSIT), 2010 3rd IEEE International Conference on , vol.5, pp.67,71, 9-11 July 2010.

[7]. Chunxi Ma, et al.; "An improved Sobel algorithm based on median filter", Institute of Electrical and Electronics Engineers, 2nd International IEEE conference, China, Volume: 1, pp. 88-93, Aug 1, 2010.

[8]. Klepper B, Browning VD, Taylor HM. "Stem diameter in relation to plant water status",PlantPhysiology,1971(48) 683-685.

[9]. S.O.Link, M.E.Thiede and M.G.van Bavel, "An improved strain-gauge device for continuous field measurement of stem and fruit diameter," Journal of Experimental Botony, Vol.49, No. 326, pp. 1583- 1587. September 1998.

[10]. Mcburney T, Costigan PA. "The relationship between stem diameter and water potentials in stems of young cabbage plants", Journal of Experimental Botany, 1984(35)1787-1793.

[11]. C.Y. Liao, V.V. Podrazsky, and G. B. Liu, "Diameter and height growth analysis for individual white pine trees in the area of Kostelec nad Cernymilesy," Journal of Forest Science, vol. 49, pp. 544-551, 2003.

[12]. T. Kärki, M. Maltamo, and K. Eerikäinen, "Diameter distribution, stem volume and stem quality

[13]. models for grey alder (Alnus incana) in eastern Finland," New Forests, vol. 20, pp. 65-86, 2000.

[14]. X.H. Sang, Y.Y. Wu, Y.G. Zhao, and M.Y. Xu, "Comparison of growth of four types of Atractylodes Lancea (Thunb.) DC," Journal of Anhui Agricultural Sciences, vol. 36, pp. 7726 - 7727, 2008. 